\title{
Clinical and Echographic Long-Term Follow-Up of a Retinal Macrocyst: A Case Report
}

\author{
Juan Carlos Serna-Ojeda Christian Denisse Pinkus-Herrera \\ Maria Victoria Moreno-Londono Jose Luis Rodriguez-Loaiza \\ Maria Cristina Gonzalez-Gonzalez \\ Instituto de Oftalmologia 'Conde de Valenciana', Mexico City, Mexico
}

\section{Key Words}

Retinal macrocyst $\cdot$ Retinal detachment $\cdot$ B-mode echography $\cdot$ A-mode echography

\begin{abstract}
The purpose of this paper is to report the case of a 62-year-old male diagnosed with a retinal macrocyst secondary to a long-standing retinal detachment in his right eye. At fundoscopy examination, an oval, elevated retinal lesion in the superior nasal quadrant was noted. Ultrasonography was performed, with a B-mode echography showing an oval, anechoic image and a standardized A-mode echography with a reflectivity spike higher than $98 \%$, which was compatible with a retinal macrocyst. The patient refused surgical treatment for the retinal detachment and was followed for 14 months with stable visual acuity and no clinical or echographic changes.

(c) 2014 S. Karger AG, Basel
\end{abstract}

\section{Introduction}

Retinal macrocysts may develop after long-standing retinal detachment. They are infrequent and most of them are generally asymptomatic, require no specific treatment and spontaneously resolve after retinal reattachment [1].

We herein report the case of a patient with a retinal macrocyst that was followed clinically and echographically for over 1 year and present a brief literature review of this clinical entity.

Juan Carlos Serna-Ojeda, MD

Instituto de Oftalmologia 'Conde de Valenciana'

Chimalpopoca 14

Mexico City 06800 (Mexico)

E-Mail juanc.sernao@gmail.com 
Serna-Ojeda et al.: Clinical and Echographic Long-Term Follow-Up of a Retinal Macrocyst: A Case Report

\section{Case Presentation}

A 62-year-old male with no comorbidities or previous ocular pathology examined in November 2012 complained of progressive blurred vision in his right eye over the past 5 years. The patient was known only for mild myopia and denied any history of previous ocular trauma.

Visual acuity was 20/60 in the right eye and 20/30 in the left eye, with a refractive error of -0.50 spherical in both eyes. Anterior segment examination of the right eye revealed nuclear and posterior lens opacities and incipient lens opacity of the left eye. No signs of previous trauma like angle recession, inferior angle pigmentation or phacodonesis were evident.

At fundoscopy examination, an oval, elevated retinal lesion in the superior nasal quadrant was noted, involving four clock hours, with no macular involvement (fig. 1). No retinal breaks were identified, with a demarcation line bordering the lesion and with the retina slightly thinned. Left fundoscopy examination was completely normal.

A B-mode superior transverse nasal echography was performed with a $10-\mathrm{MHz}$ probe. An oval, anechoic image was observed, compatible with a retinal macrocyst with smooth and well-defined borders (fig. 2a). Adjacent to the retinal macrocyst, there was a serous retinal detachment. A standardized A-mode echography showed a reflectivity spike higher than $98 \%$ (fig. 2b), thus confirming its retinal origin.

Combined scleral buckle and vitrectomy was the treatment chosen for the retinal detachment. Cataract surgery at the same surgical time was suggested but rejected by the patient. He was followed clinically and by ultrasound, first at 4 months after his initial evaluation (fig. 3a). His last examination was 14 months after presentation and revealed stable visual acuity with no clinical or echographic changes (fig. $3 b$ ).

\section{Discussion}

Retinal macrocysts are associated with long-standing retinal detachments and may be formed after 3 months, but they usually require a longer duration of retinal detachment [14]. They have been reported in 1-3\% of all long-time detachments, and most of them occur in the inferior-temporal retina, although our patient's cyst was in the superior nasal quadrant. The progression of retinal macrocysts is slow, and usually they are diagnosed in late stages [1]. Occasionally, blood may be found in their interior [2].

Retinal macrocysts may be single or multiple, measuring 2 to 10 disc diameters [2]. Pischel [5] classified retinal cysts into small (up to $1 \mathrm{~mm}$ in size), medium (4 to 8 disc diameters), giant (8 to 10 disc diameters) and large cysts (retinoschisis) if they occupy $25 \%$ of the fundus [2]. The pathogenesis has been attributed to photoreceptor atrophy due to choroid ischemia and degenerative cystic changes in the external plexiform layer of the retina that lead to cyst formation [1].

Differential diagnosis should include retinoschisis, malignant melanoma, circumscribed choroidal hemangioma, subretinal abscess and posterior scleritis; ultrasound may help in their differentiation [2]. In the case of retinoschisis, echographic findings include the lack of mobility, a thin wall and often, bilateral involvement.

Although retinal macrocysts usually resolve after treatment of the retinal detachment, they can be drained if they prevent reattachment [3]. In the case presented herein, the reduction in visual acuity was attributed mostly to the cataract, with the proposed surgery 


\begin{tabular}{l|l}
\hline Case Rep Ophthalmol 2014;5:168-171 \\
\hline DOI: 10.1159/000363759 & $\begin{array}{l}\text { ○ 2014 S. Karger AG, Basel } \\
\text { www.karger.com/cop }\end{array}$ \\
\hline
\end{tabular}

Serna-Ojeda et al:: Clinical and Echographic Long-Term Follow-Up of a Retinal Macrocyst: A Case Report

rejected by the patient. He was followed clinically and echographically for more than 1 year, and neither the macrocyst nor the serous retinal detachment progressed.

\section{Disclosure Statement}

The authors declare that they have no conflicts of interest.

\section{References}

1 Patil BB, Mowatt L, Ho S, Scott RA, Siddiqi R: Asymptomatic bilateral simultaneous rhegmatogenous retinal detachments. Eye (Lond) 2005; 19:820-821.

-2 Rishi P, Rishi E, Sen PR, Sharma T: Hemorrhagic intraretinal macrocyst: differential diagnoses and report of an unusual case. Oman J Ophthalmol 2001;4:28-31.

-3 Mendis KR, Hewick SA: Internal drainage of a retinal macrocyst with an Nd:YAG laser to aid primary retinal reattachment. Arch Ophthalmol 2009;127:791-792.

4 Mansour AM, Jaroudi MO: Hemorrhagic retinal macrocysts, simulating choroidal melanoma: a case report. Clin Ophthalmol 2013;7:973-976.

5 Pischel DK: Surgical treatment of retinal cyst. Am J Ophthalmol 1963;56:1-16.

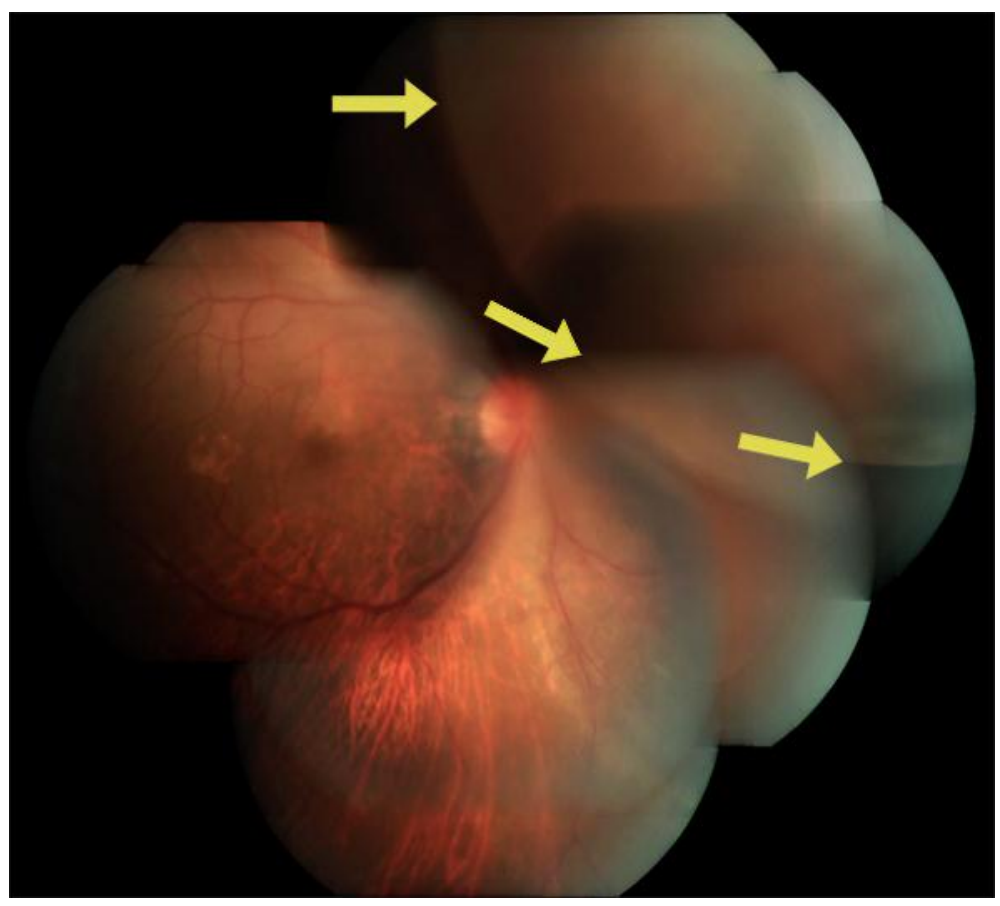

Fig. 1. Fundoscopy of the right eye, showing the lesion in the superior nasal sector. The borders of the cystic lesion are marked (arrows). 
Serna-Ojeda et al:: Clinical and Echographic Long-Term Follow-Up of a Retinal Macrocyst: A Case Report
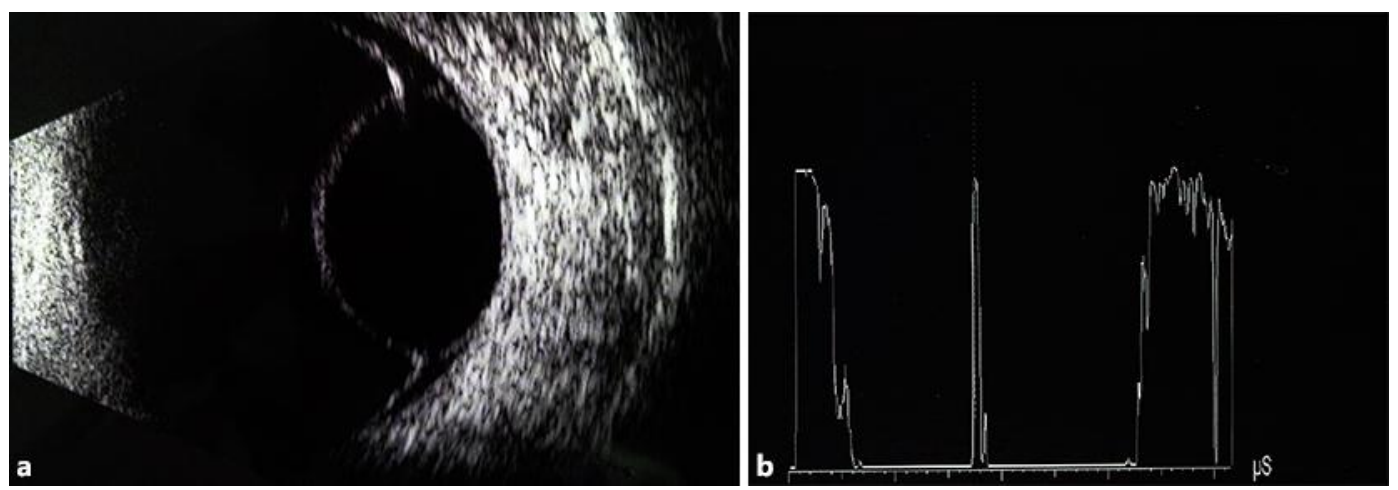

Fig. 2. a Right eye B-mode echography on patient presentation. b Right eye standardized A-mode echography.
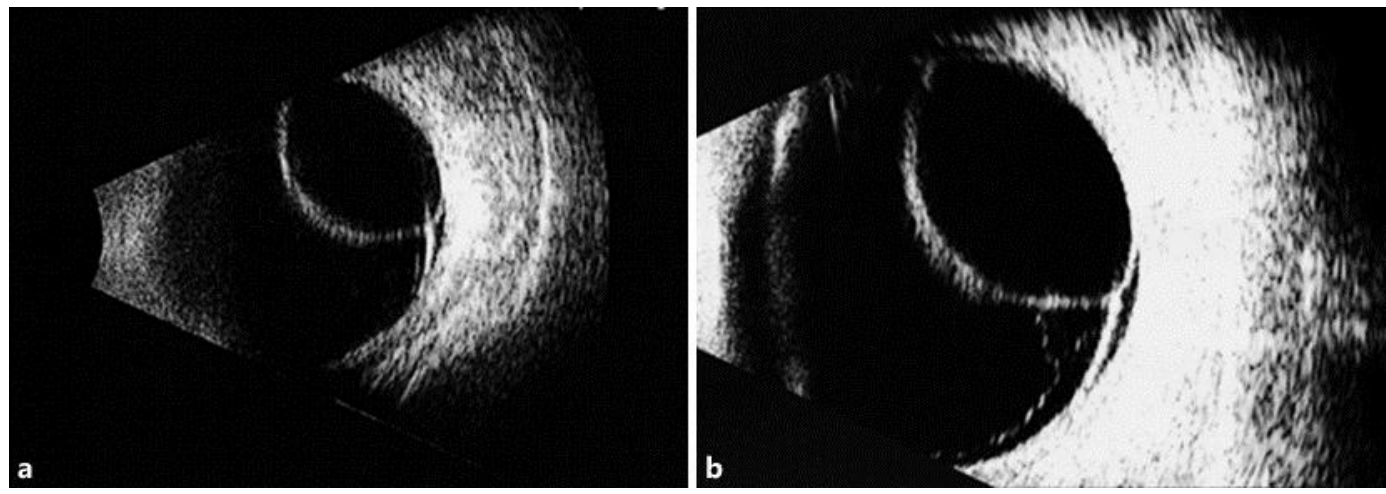

Fig. 3. a Right eye B-Mode echography with a 10-MHz probe, longitudinal axis (L2), 4 months after initial examination. b Right eye B-Mode echography with a 10-MHz probe, longitudinal axis (L2), 14 months after initial examination. 